Editorial

www.mdpi.com/journal/pharmaceutics

\title{
Acknowledgement to Reviewers of Pharmaceutics in 2014
}

Pharmaceutics Editorial Office, MDPI AG, Klybeckstrasse 64, CH-4057 Basel, Switzerland

Published: 8 January 2015

The editors of Pharmaceutics would like to express their sincere gratitude to the following reviewers for assessing manuscripts in 2014:

\begin{tabular}{|c|c|c|}
\hline Acosta, Edgar & Ensign, Laura & Liu, Fang \\
\hline Agu, Remigius & Erxleben, Andrea & Longest, P. Worth \\
\hline Alexopoulos, Aleck H & Fang, Xie & Lu, Jun \\
\hline Anadon, Arturo & Fink, James B. & Maggio, Edward T. \\
\hline Antipina, Maria N. & Frasch, H. Frederick & Mansour, Heidi M. \\
\hline Azmi, Asfar & Fresta, Massimo & Martino, Piera Di \\
\hline Babu, R. Jayachandra & Gadkar, Kapil & Mathias, Neil R. \\
\hline Beck-Broichsitter, Moritz & Garbacz, Grzegorz & McConville, Christopher \\
\hline Broutin, Sophie & Ghanotakis, Demetrios F & Milanesio, Marco \\
\hline Burley, Jonathan C. & Ham, Anthony S. & Narjoz, Celine \\
\hline Caramella, Carla & Hanada, Sanshiro & Neves, J. das \\
\hline Cevc, Gregor & Hedoux, Alain & O'Leary, Stephen \\
\hline Chatel, Jean-Marc & Hirayama, Fumitoshi & Oh, Jae-Min \\
\hline Cheung, Chi Wai & Jacobsen, Jette & Oyewumi, Moses \\
\hline Chiang, Po-Chang & Javier Gonzalez, Francisco & Papineni, Arathi \\
\hline Chiu, Loraine LY & Kalia, Yogeshvar N. & Paquette, Benoit \\
\hline Choy, Jin-Ho & Kan, Ch-Wai & Paradossi, Gaio \\
\hline Clark, Meredith & Kang, Lifeng & Park, Ji-Young \\
\hline Costantino, Luca & Kawakami, Kohsaku & Pea, Federico \\
\hline Costantino, Umberto & Kennedy, David W & Perissutti, Beatrice \\
\hline D’souza, Martin J. & Knott, R. & Petroni, Debora \\
\hline Das, Diganta B. & Kulozik, Ulrich & Rades, Thomas \\
\hline Donnelly, Ryan F. & Lam, Kim-Hung & Ribeiro, Ana C.f. \\
\hline Douglas, Timothy & Lee, Chia-Hung & Rodrigues, Alírio E. \\
\hline Douroumis, Dionysios & Leporatti, Stefano & Rohan, Lisa \\
\hline Ehrhardt, Carsten & Lin, Paulo J.C. & Rosania, Gus \\
\hline
\end{tabular}


Sahajwalla, Chandrahas G.

Stewart, Clinton F.

Su, Nan-Wei

Tang, De-Chu

Tho, Ingunn

Tian, Furong

van den Berg, Jacob J.

Vanić, Zeljka
Walzel, Peter

Watts, Peter

Yeo, Yoon

Zhou, Yue

Zumbuehl, Andreas

Shahzad, Yasser

Sparen, Anders

(C) 2015 by the authors; licensee MDPI, Basel, Switzerland. This article is an open access article distributed under the terms and conditions of the Creative Commons Attribution license (http://creativecommons.org/licenses/by/4.0/). 